\title{
Supplement to: Faster decline and higher variability in the sea ice thickness of the marginal Arctic seas
}

\author{
Robbie D.C. Mallett ${ }^{1}$, Julienne C. Stroeve ${ }^{1,2,3}$, Michel Tsamados ${ }^{1}$, Jack C. Landy ${ }^{4}$, Rosemary \\ Willatt ${ }^{1}$, Vishnu Nandan ${ }^{3}$, and Glen E. Liston ${ }^{5}$ \\ ${ }^{1}$ Centre for Polar Observation and Modelling, Earth Sciences, University College London, London, UK \\ ${ }^{2}$ National Snow and Ice Data Center, University of Colorado, Boulder, CO, USA \\ ${ }^{3}$ Centre for Earth Observation Science, University of Manitoba, Winnipeg, Canada \\ ${ }^{4}$ School of Geographical Sciences, University of Bristol, Bristol, UK \\ ${ }^{5}$ Cooperative Institute for Research in the Atmosphere, Colorado State University, Fort Collins, CO, USA
}

S1 Snow's impact on conventional sea ice thickness retrievals can be characterised solely by its snow water equivalent

Using the expression of ice freeboard from Armitage and Ridout (2015):

Ice Freeboard = Radar Freeboard + Propagation Correction

And using the expression of the propagation correction from Tilling et al. (2018):

$5 \quad h_{i}=h_{r}+h_{s}\left(c / c_{s}-1\right)$

Where $h_{s}$ is snow depth, $c$ is the speed of light in free space and $c_{s}$ is the speed of light in snow. Numerous empirical expressions for $c_{s}$ exist, in this work we use the expression for the permittivity of dry snow from Mätzler (2006):

$\epsilon_{d s}=\left(1+0.5194 \rho_{s}\right)^{3}$

Relating the radar wave speed to the permittivity using $c_{s}=c / \sqrt{\epsilon}$ (Ulaby and Long, 2014):

$10 c_{s}=c\left(1+0.5194 \rho_{s}\right)^{-3 / 2}$

The conversion of $h_{i}$ to SIT then invokes the floe's hydrostatic equilibrium and Archimedes' principle. Like the freeboard correction for slower radar pulse propagation in snow, this operation requires a priori knowledge of the depth and density of the snow cover.

$S I T=h_{i} \frac{\rho_{w}}{\rho_{w}-\rho_{i}}+h_{s} \frac{\rho_{s}}{\rho_{w}-\rho_{i}} \quad$ (Tilling et al. 2018) 
15 Separating $h_{i}$ into its $h_{r}$ and $\delta h_{\text {prop }}$ components using Eq. (S2), we can express SIT for a given ice type as a linear combination of the radar freeboard and snow properties.

$S I T=h_{r} \frac{\rho_{w}}{\rho_{w}-\rho_{i}}+h_{s} \frac{\rho_{w}}{\rho_{w}-\rho_{i}}\left[\frac{c}{c_{s}}-1\right]+h_{s} \frac{\rho_{s}}{\rho_{w}-\rho_{i}}$

$S I T=h_{r} \frac{\rho_{w}}{\rho_{w}-\rho_{i}}+h_{s} \frac{\rho_{w}}{\rho_{w}-\rho_{i}}\left(\left[\frac{c}{c_{s}}-1\right]+\frac{\rho_{s}}{\rho_{w}}\right)$

The equation $y=c / c_{s}-1$ where $c_{s}$ is a function of $\rho_{s}$ as in Eq (S4) is highly linear as a function of $\rho_{s}$ as follows:

$20 \frac{c}{c_{s}}-1=8.36 \times 10^{-4} \times \rho_{s}$

This allows the second term in Eq. (S7) to be written to a close approximation:

$$
S I T=h_{r} \frac{\rho_{w}}{\rho_{w}-\rho_{i}}+m_{s} \frac{\rho_{w}}{\rho_{w}-\rho_{i}}\left(\left(8.36 \times 10^{-4}\right)+\frac{1}{\rho_{w}}\right)
$$

Where $m_{s}$ represents the mass of snow per unit area. This can be reformulated as:

$S I T=h_{r} \frac{\rho_{w}}{\rho_{w}-\rho_{i}}+m_{s} \frac{\rho_{w}}{\rho_{w}-\rho_{i}} \times 1.81 \times 10^{-3}$ 


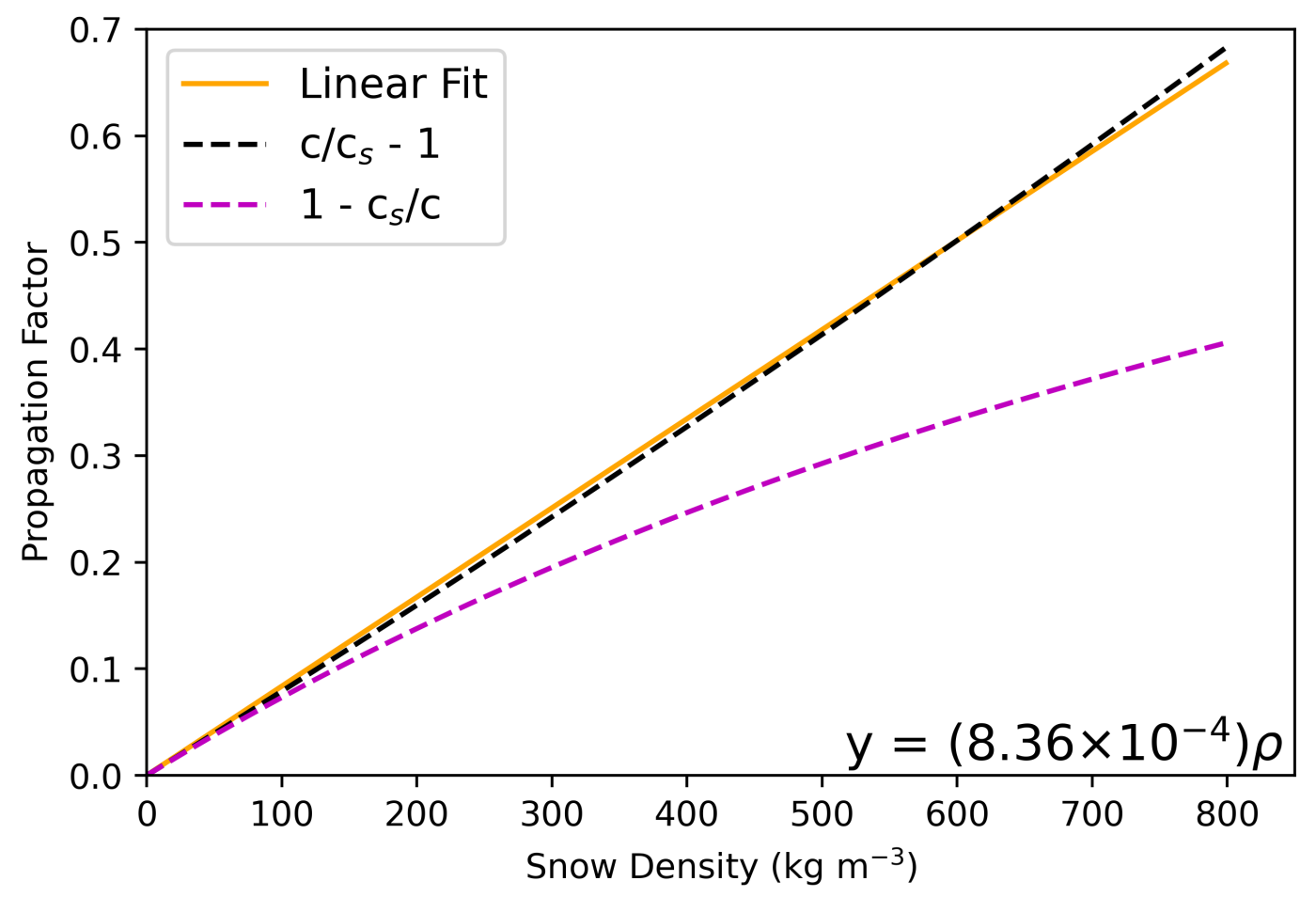

Figure S1. Value of the propagation factor used to convert radar freeboard to ice freeboard, plotted as a function of snow density. This function is highly linear and is approximated as such in this work. The factor is multiplied by the snow depth to generate the total correction. 

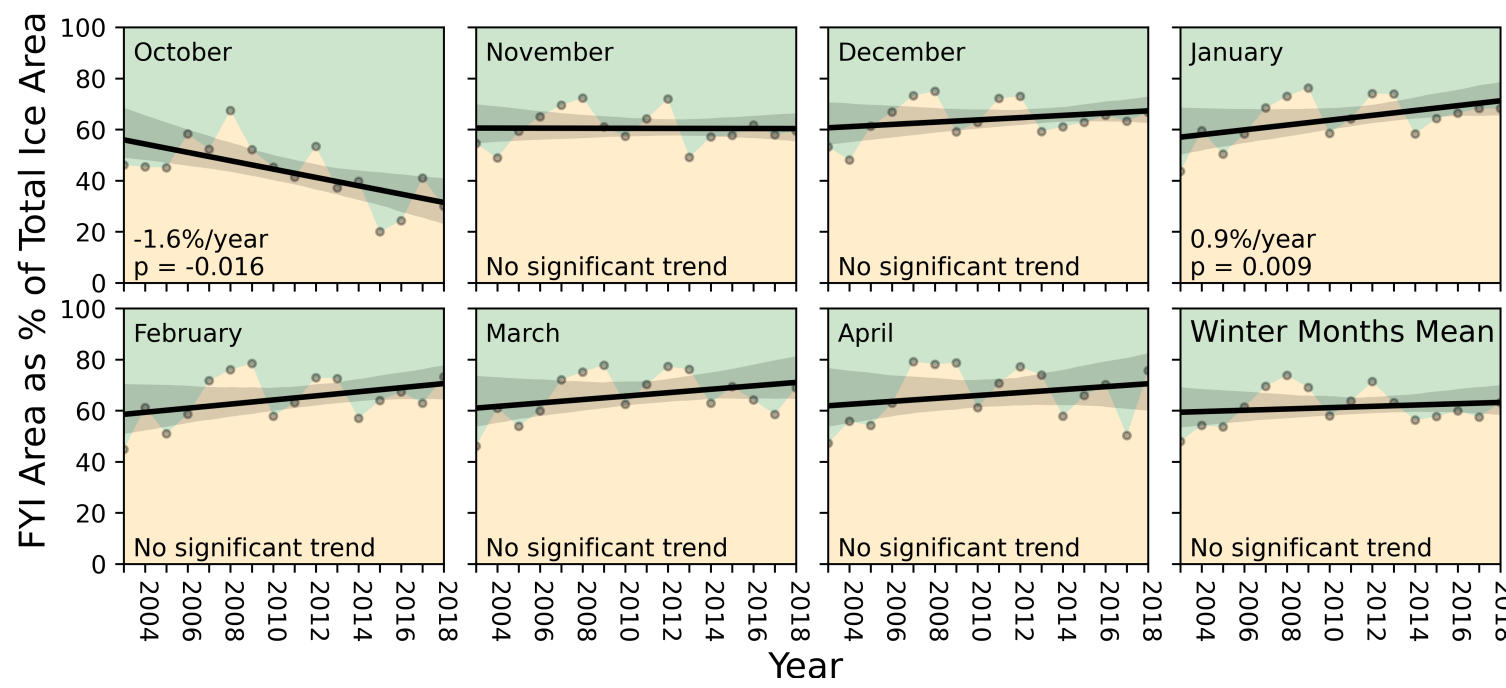

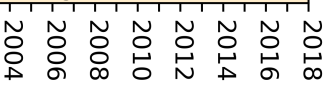

Figure S2. Basinwide trends in first year ice extent as a fraction of total extent from 2003-2018. Statistically significant trends exist in October (declining) and January (increasing). When trends of any significance are considered, all months show positive slopes barring October, which shows distinct decline. The October trend is due to later freeze-ups, the other positive trends fit in with established trends of increasing FYI dominance. Shaded regions represent the $95 \%$ confidence level for the linear regression.

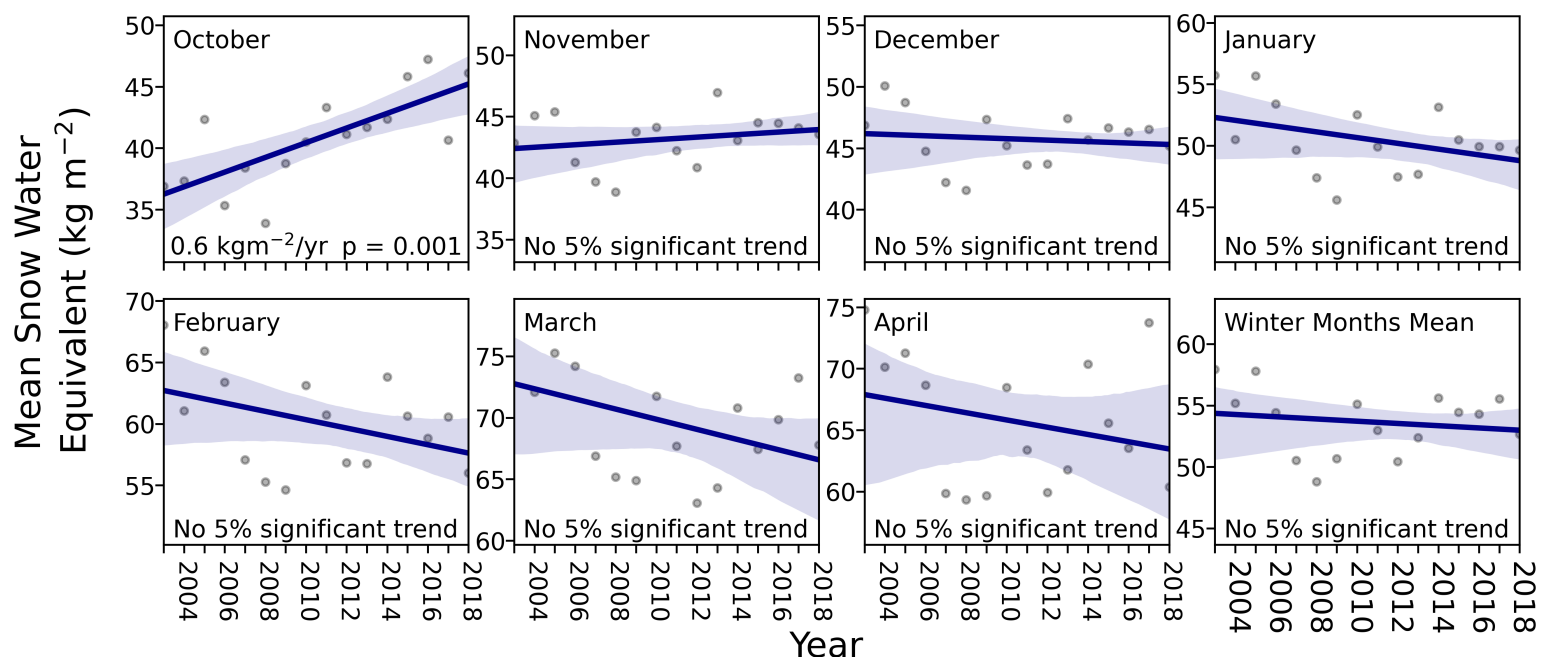

Figure S3. Basinwide trends in mW99 SWE fields from 2003-2018. A statistically significant trend only exists in October, where SWE is increasing due to the increasing dominance of MYI in the month due to later freeze-ups. Shaded regions represent the $95 \%$ confidence level for the linear regression. 


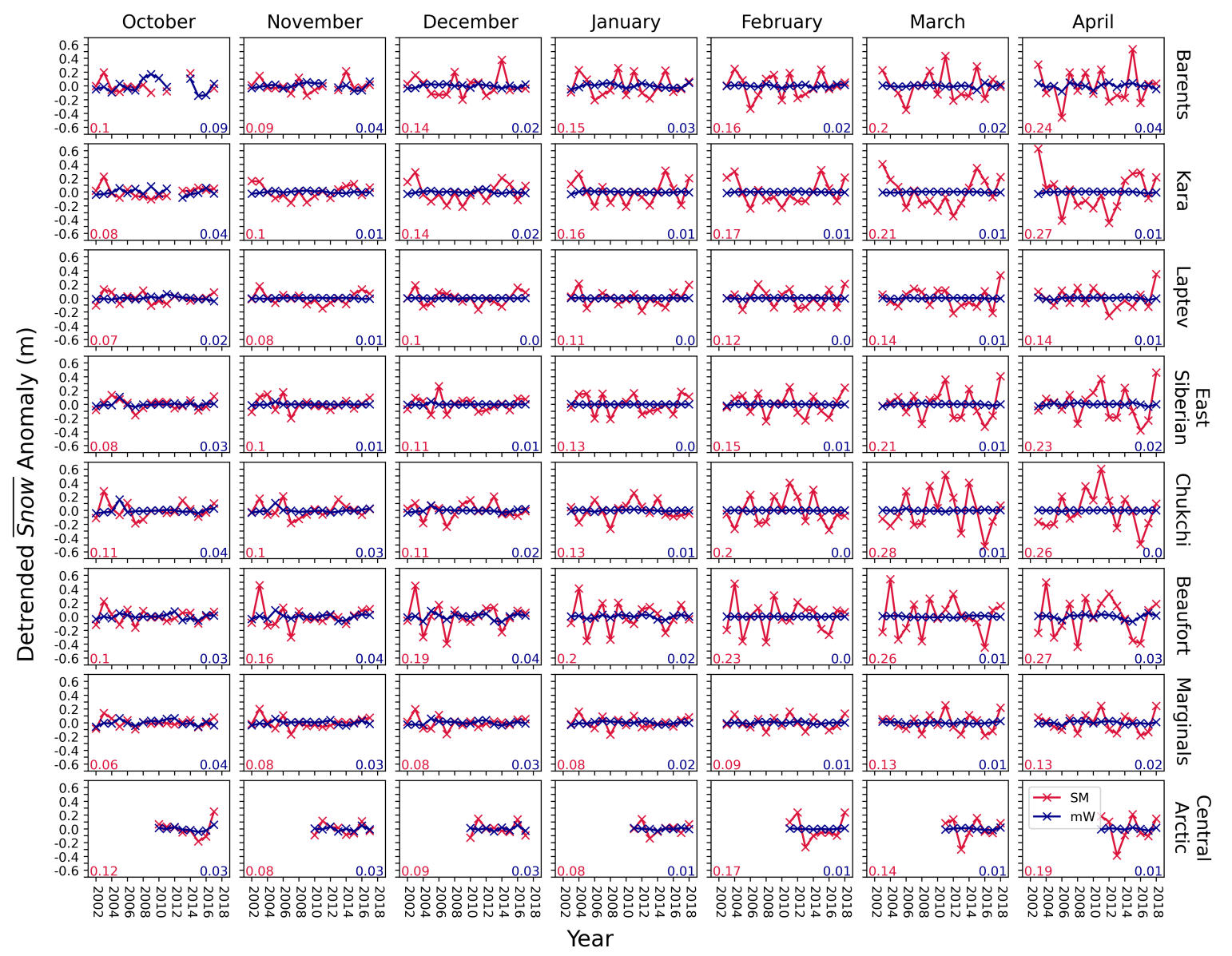

Figure S4. Detrended timeseries of spatially averaged snow contribution to sea ice thickness $(\overline{\text { Snow }})$ from W99 (blue) and SnowModelLG (red) over first year ice. SnowModel-LG is significantly more variable from year to year than W99, which only varies due to shifting dominance of ice types. This increased variability propagates through to sea ice thickness, but is moderated by its covariance with radar freeboard variability. The standard deviations of the two timeseries are displayed in the lower corners of each panel. 


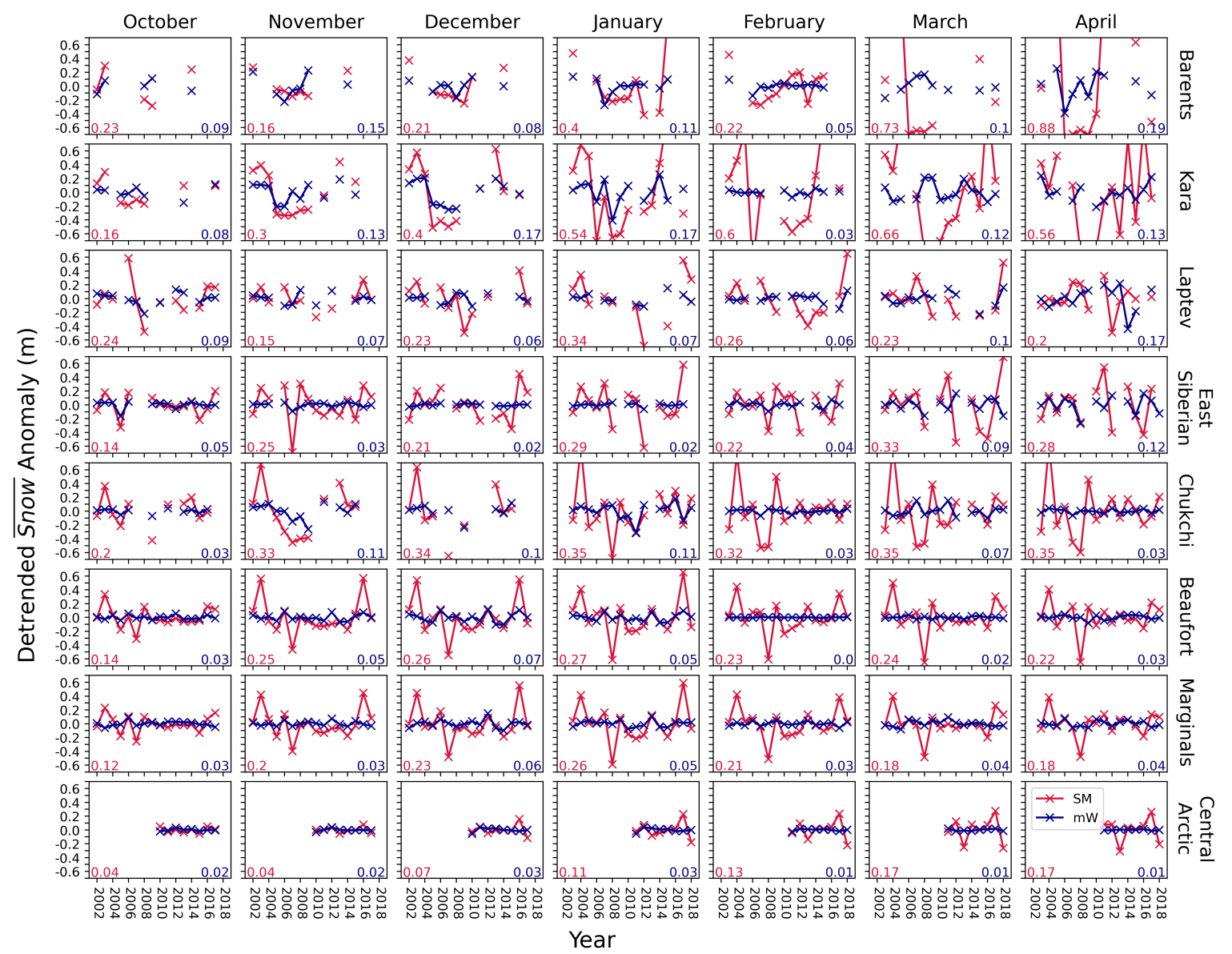

Figure S5. Detrended timeseries of spatially averaged snow contribution to sea ice thickness $(\overline{S n o w})$ from W99 (blue) and SnowModel-LG (red) over multiyear ice (MYI). SnowModel-LG is significantly more variable from year to year than W99, which only varies due to shifting dominance of ice types. This increased variability propagates through to sea ice thickness, but is moderated by its covariance with radar freeboard variability. A substantial number of data points are missing from some panels - these absences reflect months where no MYI is present in the relevant region. The standard deviations of the two timeseries are displayed in the lower corners of each panel. 


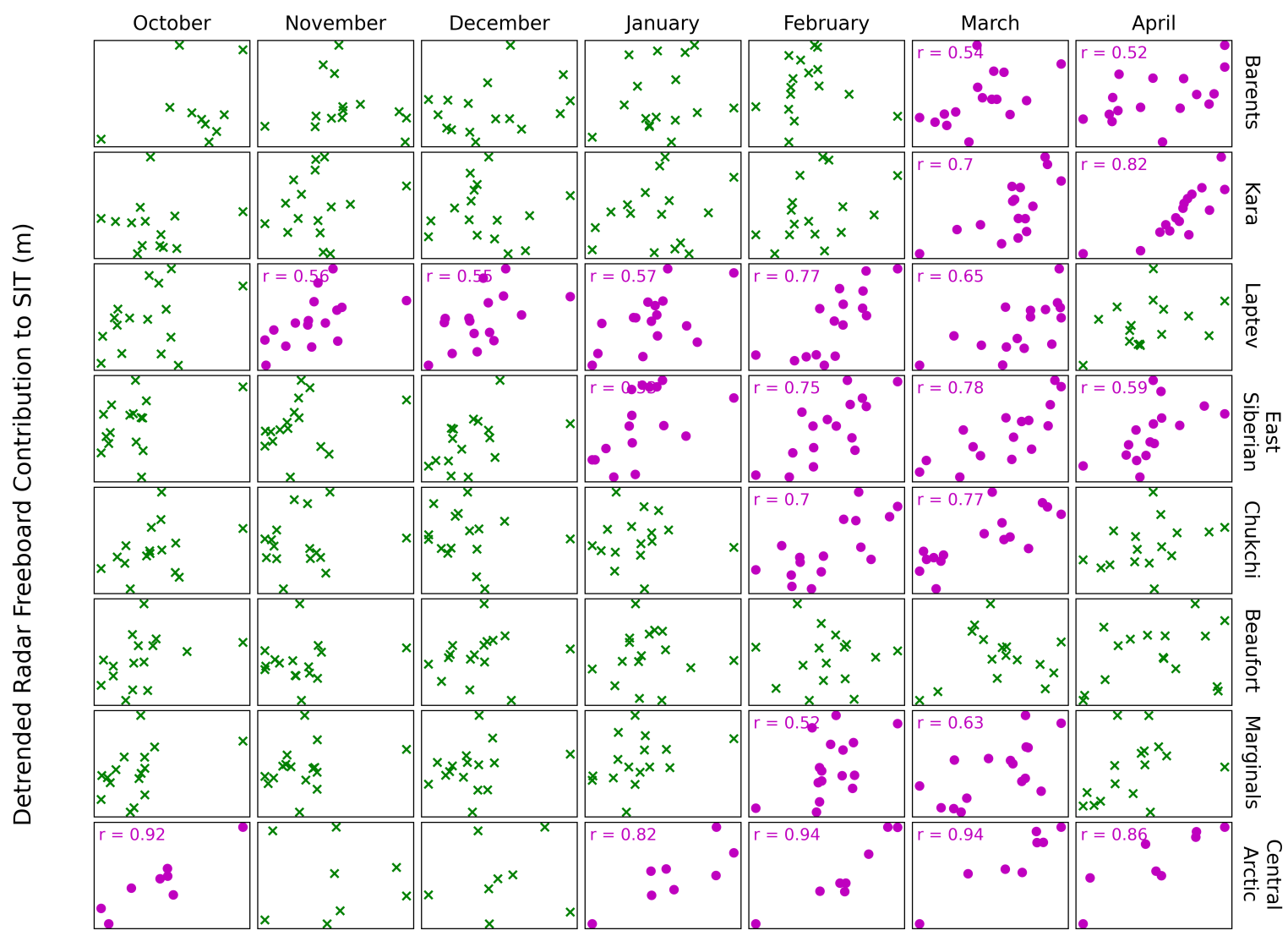

Detrended SnowModel-LG Snow Contribution to FYI SIT (m)

Figure S6. FYI correlations between radar freeboard and snow contributions to sea ice thickness, where the snow contribution is calculated using SnowModel-LG. All statistically significant correlations are positive (i.e. years with more snow exhibit higher radar freeboards). A persistent, positive correlation exists in the Central Arctic and the East Siberian Sea in the last five months of winter. The Barents and Kara Seas both exhibit significant correlations in the last two months of winter. The Beaufort sea exhibits no months of statistically significant correlation between radar freeboard and snow contributions. 


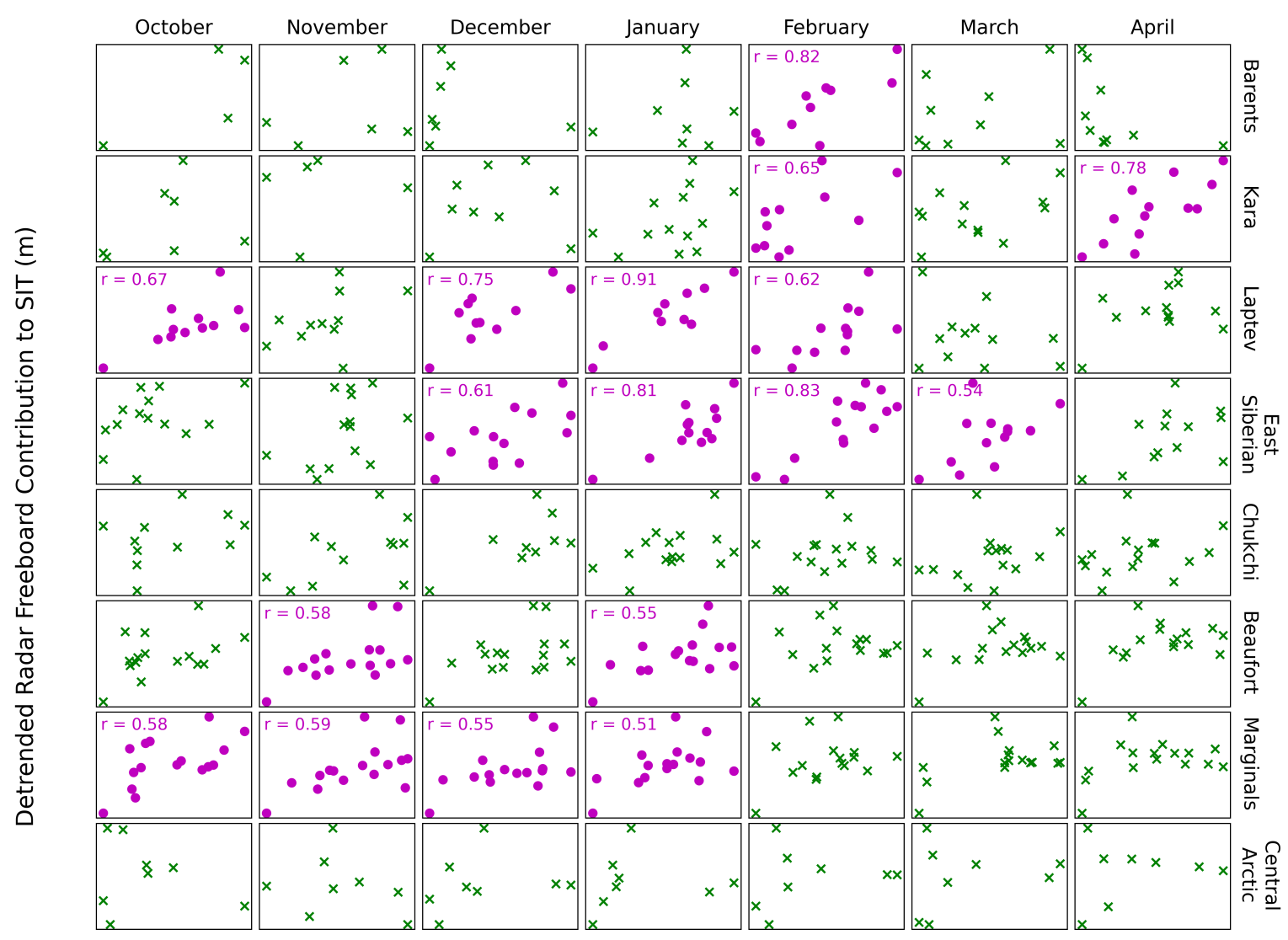

Detrended SnowModel-LG Snow Contribution to MYI SIT (m)

Figure S7. MYI correlations between radar freeboard and snow contributions to sea ice thickness, where the snow contribution is calculated using SnowModel-LG. Fewer correlations exist for MYI than for FYI. The Central Arctic and Chukchi Sea exhibit no correlations between snow and radar freeboard contributions. 
(a)

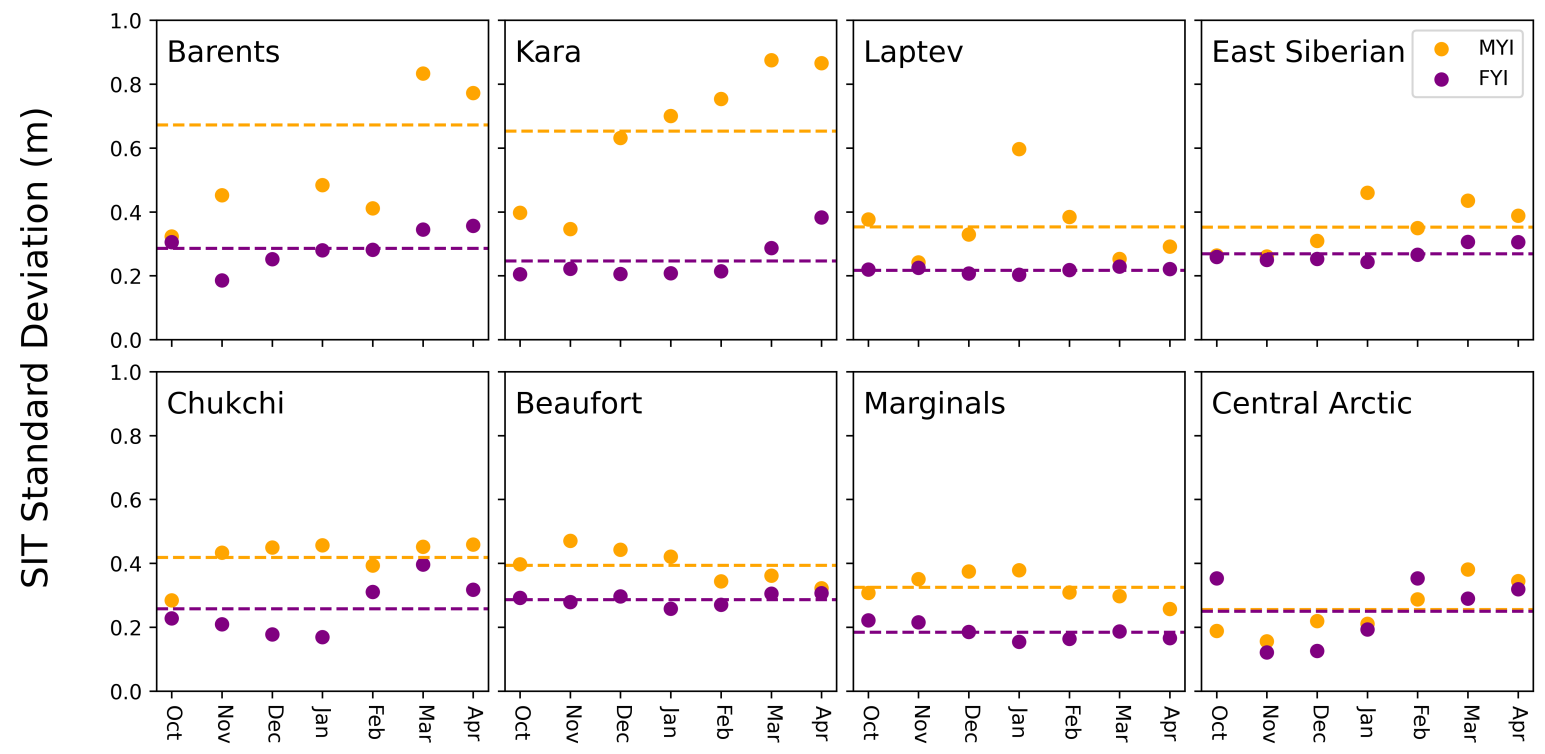

(b)
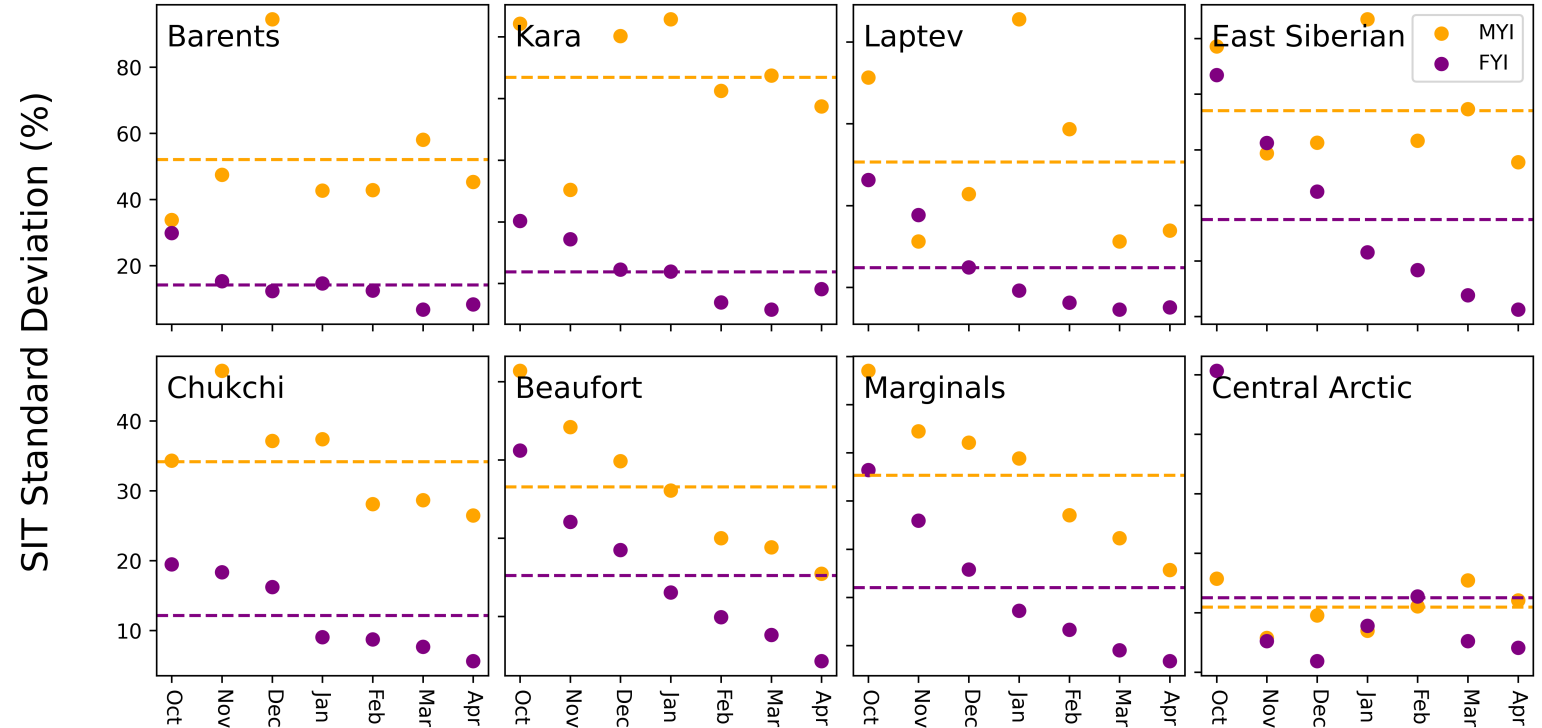

Figure S8. Regional IAV displayed by ice type. MYI represented by orange points, FYI represented by purple. When averaging over the growth season in a given region, MYI is more variable in all the marginal seas. 


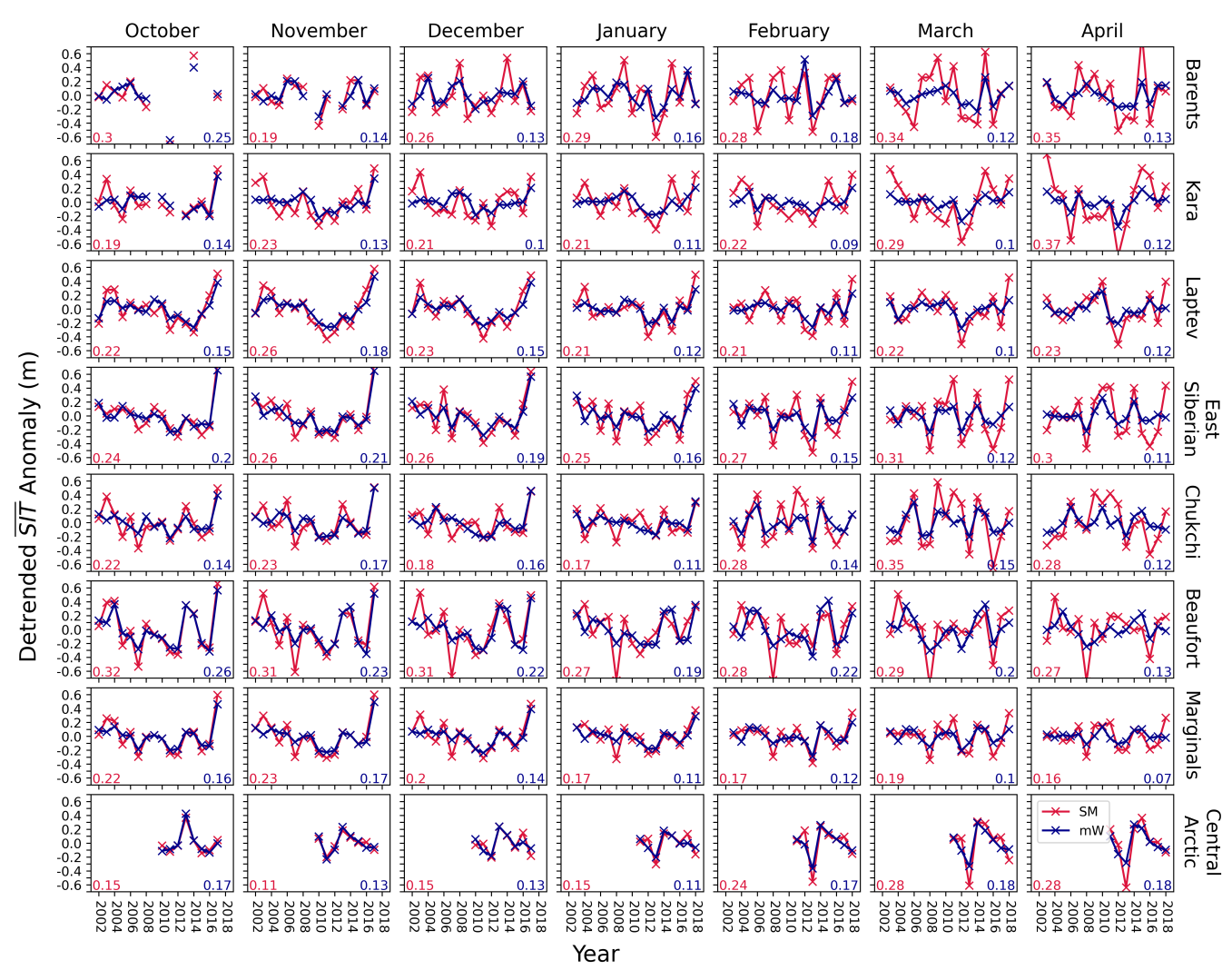

Figure S9. Detrended timeseries of spatially averaged sea ice thickness $(\overline{S I T})$ by region from W99 (blue) and SnowModel-LG (red) for all ice types. Standard deviation values are displayed for SnowModel-LG (lower left, red), and mW99 (lower right, blue). 


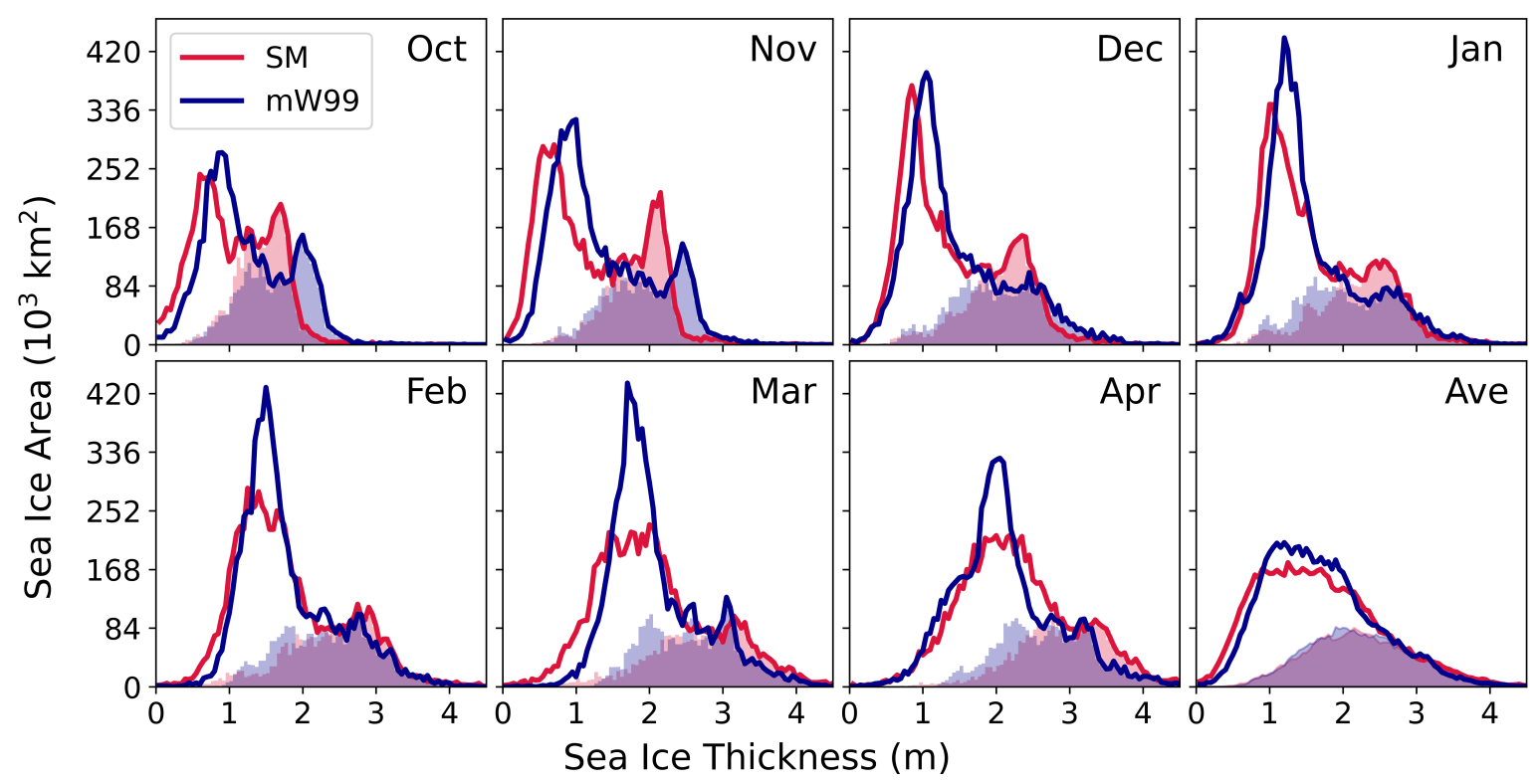

Figure S10. Basin-wide sea ice thickness distribution calculated using both mW99 and SnowModel-LG data. Shaded areas represent the area constituted by the Central Arctic. 
(b)

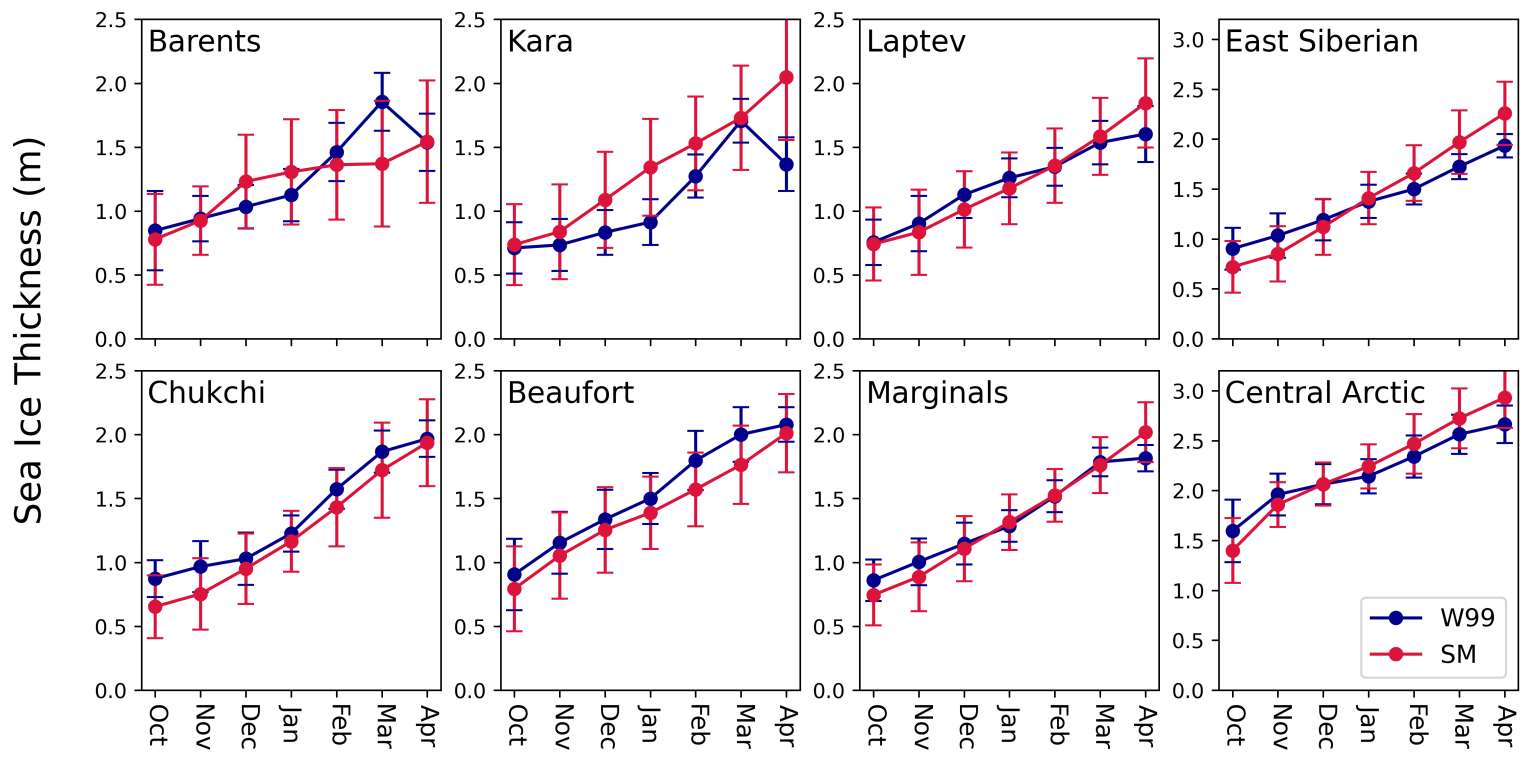

(b)
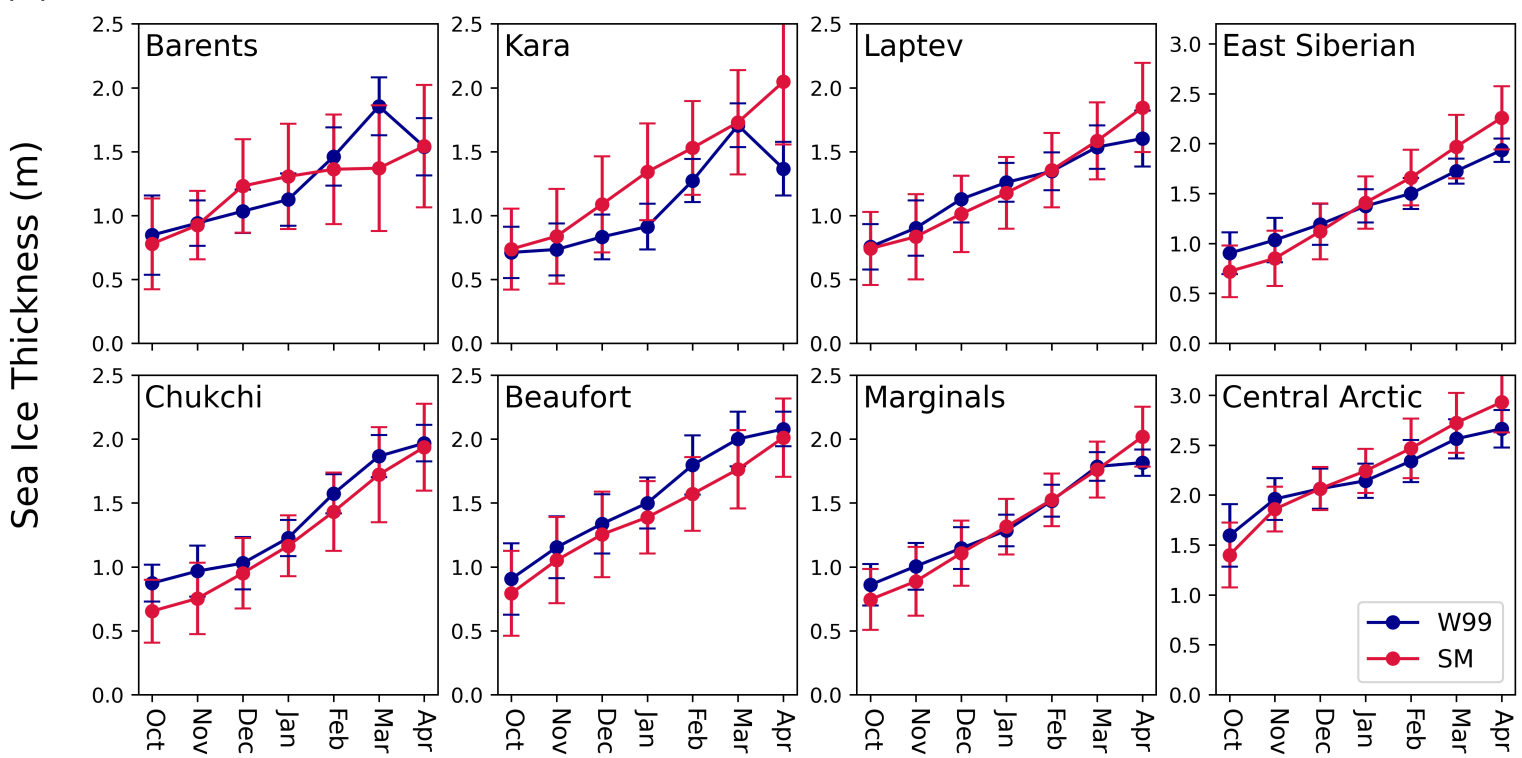

Figure S11. Seasonal evolution of sea ice thickness by region. The SnowModel-LG contribution starts lower but ends higher in the Central Arctic, the region that dominates Pan-Arctic statistics. This corresponds to faster thickness increase than would be calculated with W99. 

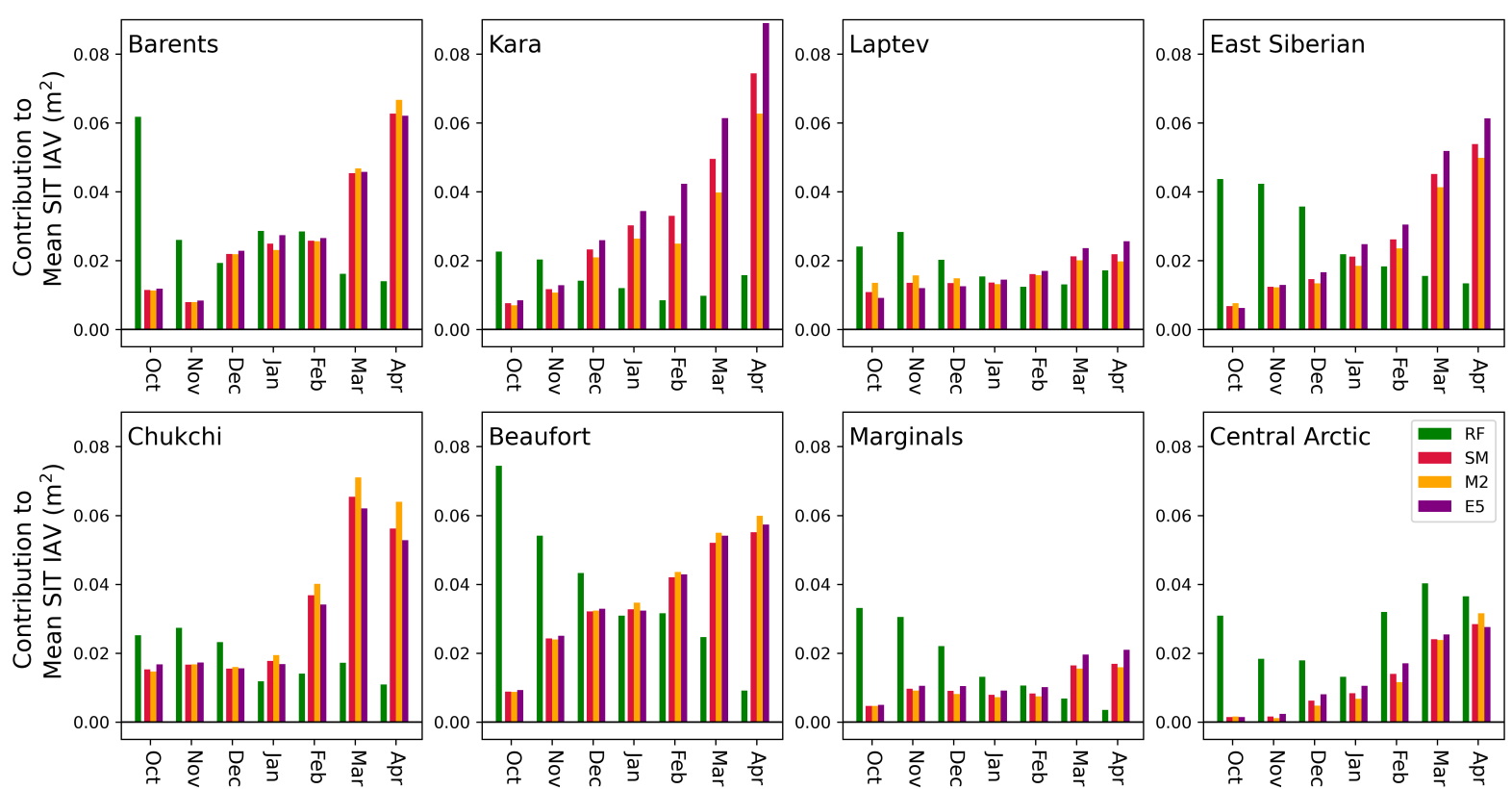

Figure S12. Interannual variability of SnowModel-LG contribution to $\sigma_{\overline{S I T}}^{2}\left(\sigma_{\overline{S n o w}}^{2}\right)$ when forced by two different reanalysis data sets. MERRA2 (orange) and ERA5 (green) produce very similar variability. 


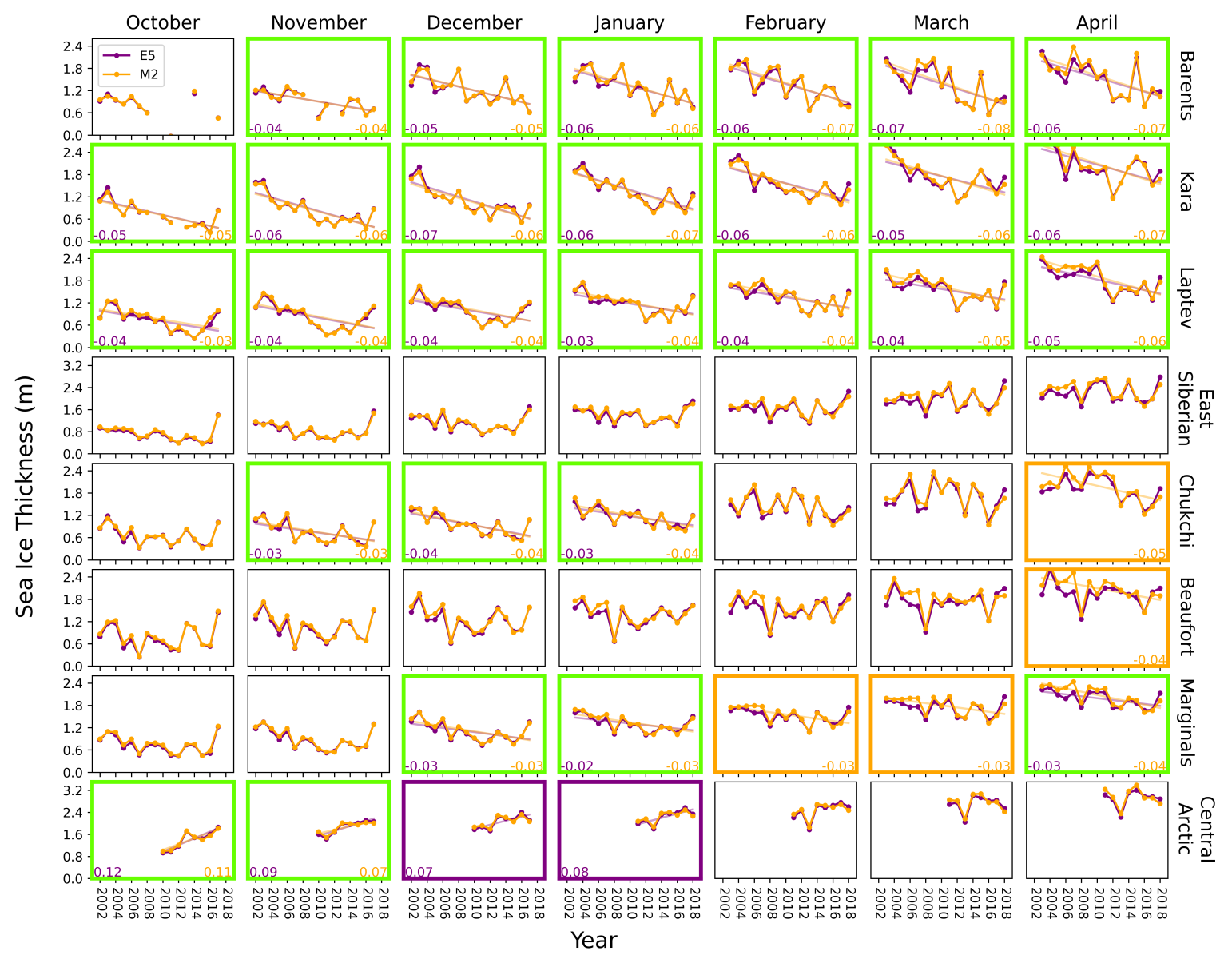

Figure S13. Trends in sea ice thickness (2002-2018) by region, when calculated using SnowModel-LG runs using two different sources of reanalysis (ERA5, Purple; MERRA2, Orange). Panels are framed with green where statistically significant trends exist independent of reanalysis choice. Purple (orange) frames represent month/region pairs where statistically significant trends are only present with ERA5 (MERRA2). Slope values are given where significant in the lower corners. All significant trends in the marginal seas are negative, all significant trends in the Central Arctic are positive. In the Central Arctic, two of the four statistically significant increasing trends are only evident with ERA5 reanalysis. In the Marginal Seas, the decline in some months is only statistically significant with MERRA2. 


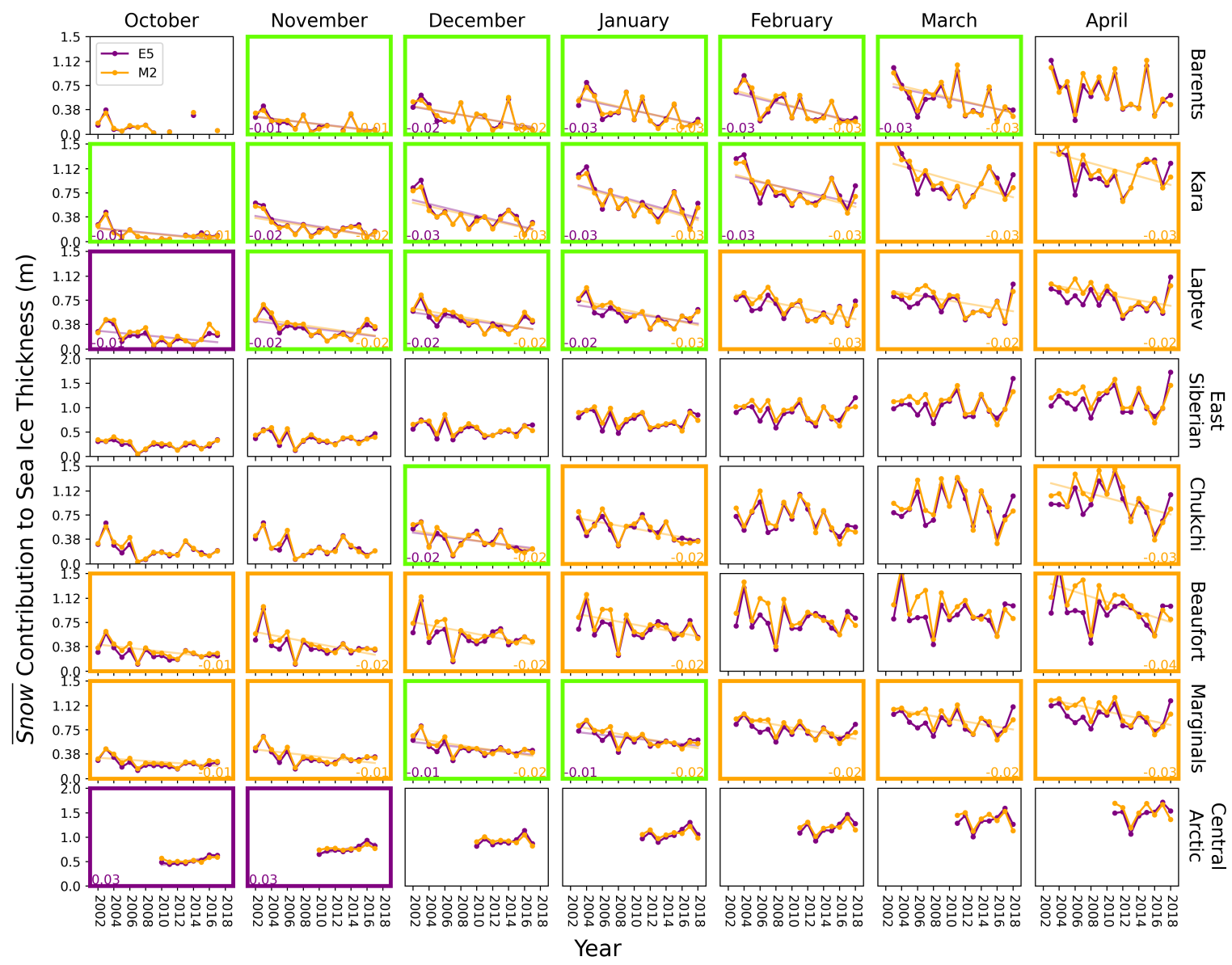

Figure S14. Trends in snow contribution to sea ice thickness $(\overline{\text { Snow }} ; 2002-2018)$ by region, when calculated using SnowModel-LG runs using two different sources of reanalysis (ERA5, Purple; MERRA2, Orange). Panels are framed with green where statistically significant trends exist independent of reanalysis choice. Purple (orange) frames represent month/region pairs where statistically significant trends are only present with ERA5 (MERRA2). Slope values are given where significant in the lower corners. 

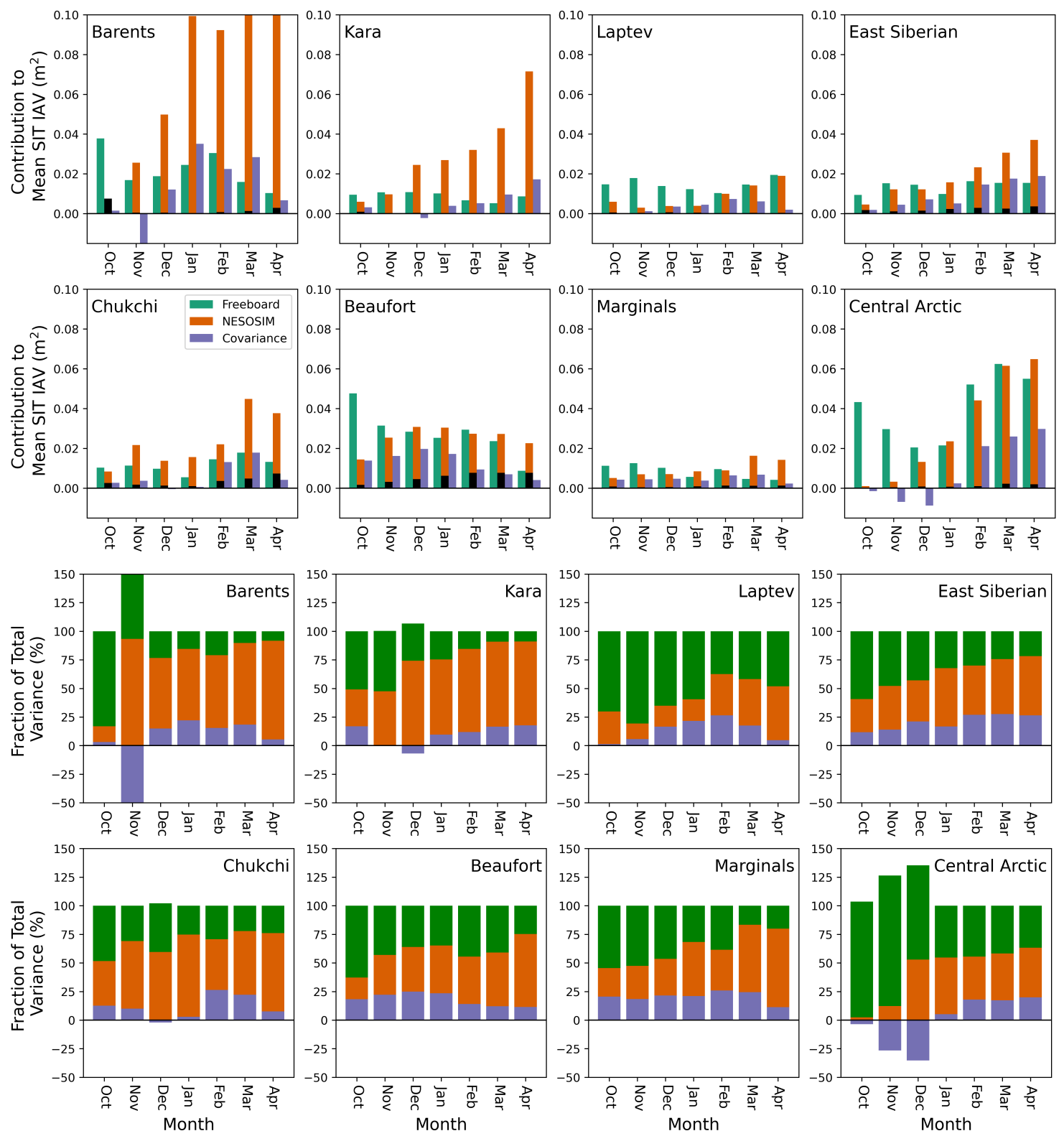

Figure S15. Interannual variability of NESOSIM data's contribution to SIT, shown as (a) absolute contribution to SIT variability, and (b) relative contribution. Variability from snow is of a similar magnitude to that of SnowModel-LG, although regional differences exist between the corresponding plots, particularly in the Barents Sea. As well as differences in the snow accumulation scheme, the two data sets differ in spatial resolution and the timespan over which they are analysed. 


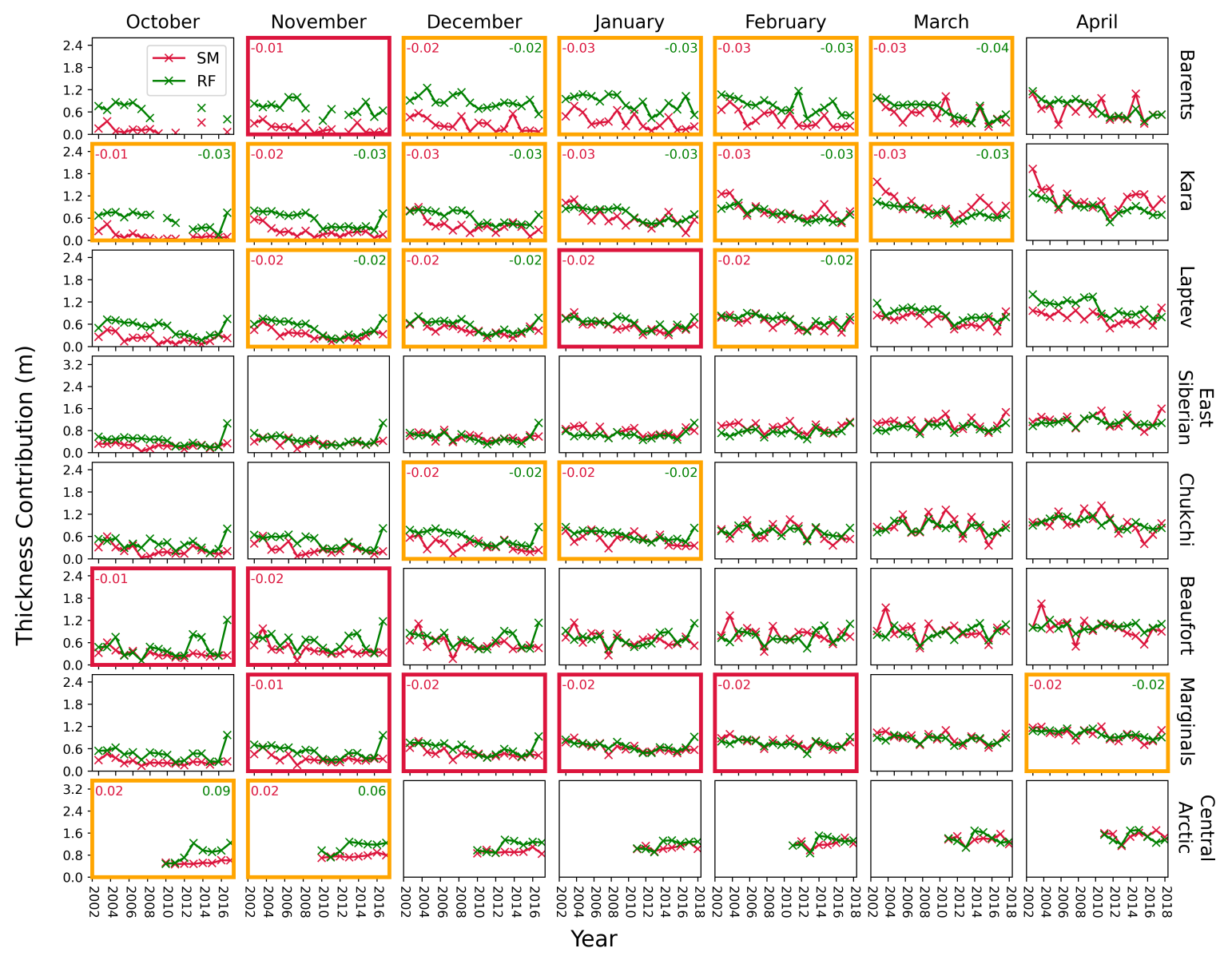

Figure S16. Timeseries of the thickness contributions of radar freeboards $(\overline{R F})$ and snow $(\overline{S n o w})$ over all ice types. Orange framed boxes indicate statistically significant decline in both $\overline{R F}$ and $\overline{S n o w}$. The red framed box indicates statistically significant decline in $\overline{\text { Snow }}$ only. No boxes feature a statistically significant decline in $\overline{R F}$ without a concomitant decline in $\overline{S n o w}$. All statistically significant trends in both $\overline{S n o w}$ and $\overline{R F}$ are negative. 


\section{References}

Armitage, T. W. and Ridout, A. L.: Arctic sea ice freeboard from AltiKa and comparison with CryoSat-2 and Operation IceBridge, Geophysical Research Letters, 42, 6724-6731, https://doi.org/10.1002/2015GL064823, 2015.

Mätzler, C.: Thermal microwave radiation: applications for remote sensing, vol. 52, Iet, 2006.

Tilling, R. L., Ridout, A., and Shepherd, A.: Estimating Arctic sea ice thickness and volume using CryoSat-2 radar altimeter data, Advances in Space Research, 62, 1203-1225, https://doi.org/10.1016/j.asr.2017.10.051, https://doi.org/10.1016/j.asr.2017.10.051, 2018.

Ulaby, F. and Long, D.: Microwave Radar and Radiometric Remote Sensing, The University of Michigan Press, https://doi.org/10.3998/0472119356, http://public.ebookcentral.proquest.com/choice/publicfullrecord.aspx?p=4537961, 2014. 